Ann. Abeille, I965, 8 (2), 95-107.

\title{
LA DUREE DE VIE DES REINES D'ABEILLES (APIS MELLIFICA MELLIFICA L.) EN CAGES D'EXPÉDITION
}

\author{
J. FRESYAIE \\ Station expérimentale d'Apiculture, \\ Centre de Recherches agronomiques du Sud-Est, Montfavet (Vaucluse)
}

SOMMAIRE

L'auteur étudie l'influence du nombre des abeilles d'accompagnement sur la durée de vie des reines en cages d'expédition. Il met en évidence l'importance du volume de la cage qui doit être proportionnel au nombre d'abeilles qui l'occupent, ainsi que l'influence de divers autres facteurs tels que la nourriture et l'hygrométrie.

\section{INTRODUCTION}

Le transport des reines d'abeilles a débuté presque en même temps que l'apiculture moderne en ruches à cadres mobiles, il $y$ a une centaine d'années. D'abord, des ruches peuplées furent seules transportées sur des distances de plus en plus longues. Puis on s'aperçut que les reines pouvaient voyager dans des conditions moins onéreuses et plus faciles qu'avec leur ruche, c'est-à-dire dans de petites cages contenant seulement quelques abeilles d'accompagnement et un pet de nourriture. Ce fut la naissance des cagettes pour le transport des reines en même temps que celle de la profession d'éleveur de reines.

Il apparut alors rapidement un très grand nombre de modèles de cages d'expédition. Le principal problème considéré dans l'élaboration de leur conception n'était d'ailleurs pas celui des conditions de voyage mais celui de l'introduction des reines après le voyage. Une reine étrangère, et surtout une reine ayant voyagé, ne peut être introduite dans une ruche sans de grandes précautions sous peine de la voir attaquée, " emballée " (1) et tuée dans la plupart des cas. C'est pourquoi, afin d'éviter des manipulations trop délicates ou trop longues, la cage de transport reçut la mission

(1) La reine étrangère se trouve entourée par un grand nombre d'ouvrières qui forment autour d'elle une a balle "; d'où le terme a emballée "utilisé par les apiculteurs. 
d'assurer l'introduction de la reine dans sa nouvelle ruche. La cage Benton fut l'une des premières utilisées en apiculture. La plupart des autres cages d'introduction ne sont, en fait, que des variantes, plus ou moins compliquées, du modèle Benton. Elle garde d'ailleurs la faveur de la majorité des éleveurs de reines en raison de son bas prix de revient, de sa simplicité, et d'un pourcentage d'introductions réussies qui égale approximativement celui des autres cages basées sur le même principe.

İn I.940, SNEIGROVE publiait un répertoire complet des différentes cages et méthodes d'introduction des reines et faisait le point des connaissances à ce sujet.

Plus récemment, différents auteurs contribuèrent, à l'étude des conditions de vie des reines et des abeilles ouvrières en cages.

Woodrow (1938, I94I) compare la durée de rie de reines en cages avec apport d'eau et sans eau, dans des étuves aỹant des températures de $28^{\circ}$ à $30^{\circ}$ et $\mathrm{I} 9$ à 66 p. Ioo d'humidité relative. Il trouve que 93 p. Ioo des reines, nourries avec du " candi à reines " (miel et sucre en poudre, malaxés pour former une pâte très ferme), pourvues d'eau accessible dans de petits flacons, vivent plus de deux semaines. Par contre, Io p. Ioo seulement des reines nourries au candi seul, sans apport d'eau, vivent plus de 5 jours.

I'après MAURizio (1946), l'approvisionnement en eau et la température sont des facteurs capitaux. Des abeilles encagées, privées d'eau, vivent trois à quatre fois moins longtemps que celles qui sont bien abreuvées. I'humidité de l'air est également un facteur très important. La survie n'excède pas 8,4 jours à $97 \mathrm{p}$. Ioo d'humidité pour atteindre 35,2 jours à 25 p. IOO. Maurizıo trouve également de grandes différences entre abeilles d'été et abeilles d'hiver; les premières vivaient en moyenne 24 à 25 jours, les secondes 36 à 37 jours (chiff res voisins de ceux de FREE et SPEXCER BOOTH (I959). Rocksterr (I 950) trouve une moyenne de vie de $5 \mathrm{r}$ jours; les abeilles encagées vivraient 2 à 3 semaines de plus. Un facteur très important a été étudié par Chauvin et Grassí (I944) et Chauvis (I95I). Il s'agit de l'effet de groupe, lequel est particulièrement important chez l'abeille. Celle-ci meurt très rapidement, en $\delta$ jours au plus, lorsqu'elle est isolée, même si elle est maintenue dans des conditions physiques identiques à celles des groupes. Pour obtenir une bonne survie il faut dépasser 30 abeilles par cage. Ce nombre minimum de 30 abeilles se retrouve également dans l'étude sur le thermopréférendum de l'abeille faite par IAVIE et ROTH (1953).

KHARCHEVA (I957) maintient des reines dans de petites cages avec Io abeilles pendant 17 à 46 jours; elles sont nourries au miel ; les abeilles mortes sont enlevées périodiquement et remplacées par de jeunes abeilles. L'addition de ces nouvelles abeilles provoque l'augmentation temporaire du poids des reines et démontre l'importance de l'état physiologique des abeilles d'accompagnement sur la santé des reines qu'elles nourrissent.

Par ailleurs, AI,LEN (I955) signale que ce sont les ouvrières âgées de I à II jours qui nourrissent la reine et que celles qui ont de I à 36 jours la lèchent.

Enfin, des expériences de I)REHER (I954) concernant les conséquences d'une claustration prolongée des reines fécondes démontrent que jusqu'à 2 jours et demi cette claustration n'a aucune conséquence grave. Mais, dès le $3^{\mathrm{e}}$ jour, l'activité de ponte ultérieure est perturbée, et à partir du sixième jour, 41 p. 100 des reines claustrées ne peuvent franchir l'hiver.

La cage Benton, ainsi que toutes les cages décrites par SLEI.GROVE, ne peuvent 
recevoir qu'un très petit nombre d'abeilles : une dizaine en général ; en raison de leur faible volume, bien souvent ce nombre n'est pas atteint.

On trouve plus fréquemment dans ces cages 5 ou 6 " accompagnatrices ". Plusieurs raisons sont à l'origine de ce volume réduit ainsi que du petit nombre d'abeilles accompagnant la reine :

a) La cage d'introduction doit pouvoir se glisser entre les rayons des ruches.

b) Son faible volume permet de grouper Io, 20 ou 50 cages et doit permettre le transport par avion et par poste pour un minimum de frais.

c) Les accompagnatrices sont très mal acceptées dans les ruches ainsi que l'ont démontré OROSI-PAL (I955) puis MORSE et G.ARY (I96I) ; à plus forte raison lorsque leur nombre est élevé.

d) Les nuclei de fécondation sont parfois peu peuplés et le prélèvement d'un certain nombre d'abeilles au profit des cages de transport risquerait de trop les affaiblir lorsqu'ils doivent servir pour de nouvelles reines.

e) J,e prélèvement des abeilles et leur introduction clans les cages se fait en les prenant une à une, généralement à l'aide d'une pince entomologique très souple dite " pince de chasse ". Ce travail est très long et rebute les éleveurs de reines.

f) Les recherches concernant la durée de vie des abeilles en cages ont, pour la plupart, été faites en étuve, donc dans des conditions très artificielles. Elles sont peu nombreuses en ce qui concerne le transport des reines et, en outre, les résultats obtenus par les chercheurs sont méconnus des apiculteurs.

Il nous a semblé que l'importance du nombre d'abeilles dans les cages de transport pour reines avait été beaucoup trop négligée jusqu’à présent. Nous présentons ici les résultats d'expériences qui montrent que nos connaissances sur la physiologie de l'abeille encagée sont susceptibles de trouver une application pratique dans le domaine du transport des reines à longue distance.

\section{MATÉRIEL ET MÉTHODE}

La cage expérimentale utilisée (fig. I) a pour dimensions $105 \mathrm{~mm}$ de long, $50 \mathrm{~mm}$ de large et $23 \mathrm{~mm}$ d'épaisseur. Les côtés et la cloison sont en bois blanc de $5 \mathrm{~mm}$ d'épaisseur, le fond en contreplaqué de $3 \mathrm{~mm}$, le dessus en grillage à mailles de $2 \mathrm{~mm}$. Le volume réservé aux abejlles

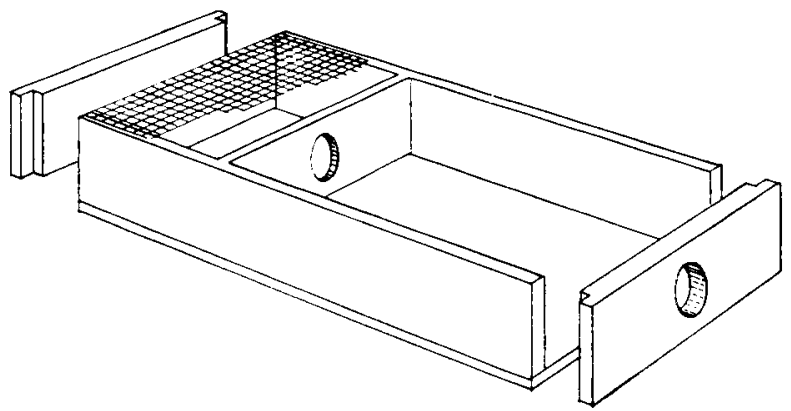

FIG. I. - Perspective cavalière de la cage expérimentale, grillage particllement enlevé, les couvercles sont ouverts montrant l'ouverture lotale de la cage, four l'entrée des abeilles d'un cóté, pour remplir le magasin d candi de l'autre.

Annales de l'Abeille. - I965. 
est ainsi de $52 \mathrm{~cm}^{3}$ et celui destiné au candi de $16 \mathrm{~cm}^{3}$ environ. Les deux côtés les moins longs forment des couvercles dont l'ouverture totale permet d'un côté d'introduire le candi et, de l'autre, de faire pénétrer les abeilles. On opère, soit en glissant la cage le long du cadre garni d'abeilles, sans appuyer, soit en faisant tomber d'une légère secousse la petite grappe d'abeilles qui se forme souvent à la base des cadres que l'on sort des ruches ou des nuclei de fécondation. Un trou de $10 \mathrm{~mm}$ de diamètre, pratiqué dans le couvercle du côté réservé aux abeilles, permet d'introduire facilenent la reine dans la cage après le chargement des abeilles.

Les cages Benton qui nous ont servi pour les comparaisons sont du type le plus courant, dont les dimensions extérieures sont de $100 \mathrm{~mm} \times 32 \mathrm{~nm} \times 22 \mathrm{~mm}$. Ce modèle de cage est mondialement connu, nous n'en donnerons donc pas de description plus détaillée.

L'eau est offerte aux abeilles dans les cages à l'aide d'un morceau de coton hydrophile imbibé d'eau, déposé sur le grillage dans la partie de la cage opposée à celle qui contient le candi. Il faut toujours prendre toutes les précautions nécessaires pour qu'il ne s'écoule aucune goutte d'eau dans la cage afin que les abeilles ne se mouillent pas ou que le candi ne fonde et les englue.

Les reines jeunes ont été prélevées dans des nuclei de fécondation, environ deux semaines après qu'elles aient commencé it pondre. Elles sont donc dans des conditions physiologiques comparables à celles des reines hat ituellement expédiées par les éleveurs.

Les reines âgées de 2 ans ont été prélevées dans des ruches destinées à la production de miel. A cet âge, elles étaient vouées à une rapide disparition et l'on ne pourra s'étonner de trouver de très grandes variations dans les résultats qu'elles fournissent.

Le candi qui sert de nourriture dans les cages est le "candi à reines " ordinaire. Il est coniposé de miel liquide auquel on incorpore, à chaud, du sucre en poudre trés fin jusqu'à saturation, de façon à obtenir une pâte très ferme.

Au cours des différentes expériences, les cagettes contenant reines et abeilles ont été placées dans une grande boite en carton, aérée mais obscure. Cette boîte et son contenu restaient soumis aux variations de température et de l'état hygrométrique de l'air au laboratoire. Au cours des expériences, la température a oscillé entre $2 \mathrm{I}^{\circ}$ et $29^{\circ}$ et l'humidité relative entre 50 et $65 \mathrm{p}$. 100 , suivant les jours et les heures. Ainsi, les conditions de vie de ces abeilles se trouvaient aussi proches que possible de celles rencontrées lors d'un transport de reines dans la plupart des cas sous notre latitude et pendant la belle saison.

\section{RÉSULTATS}

Au cours de nos expériences, nous avons étudié un certain nombre de possibilités de conditions de vie avec nos cages expérimentales et les cages Benton. Nous avons réuni les résultats dans le tableau I qui permet de comparer les divers essais. Tous les détails concernant chacune des expériences sont donnés ci-dessous :

\section{Io Cages Benton, reines jeunes, accompagnées de 10 ouvrières, sans apport d'eau}

La plupart des reines expédiées dans le monde par les éleveurs se trouvent dans des conditions de vie proches de celles de cette série. Elle nous servira donc de base de comparaison pour juger les autres essais. Cependant, en raison des résultats obtenus dans l'ensemble de nos expériences, nous devons considérer que les conditions de survie de cette première série sont meilleures que celles rencontrées habituellement car, bien souvent, 5 ou 6 ouvrières seulement accompagnent les reines. La durée de survie des reines de cette série est comprise entre I 2 et 27 jours; la durée moyenne pour la série est de 18,5 jours.

$$
\begin{aligned}
& 2^{\circ} \text { Cages Benton, reines jeunes, } \\
& \text { accompagnées de } 10 \text { ouvrières, avec apport d'ean }
\end{aligned}
$$

L'apport d'eau est pratiqué comme précédemment décrit. A la suite des travaux antérieurs déjà cités, nous pensions obtenir une augmentation notable de la durée 
de vie des reines et des abeilles de cette série. A notre grande surprise, la durée de vie moyenne fut seulement de I2,6 jours avec des extrêmes de 8 et I9 jours. Nous retrouverons d'ailleurs cette différence dans les séries réalisées avec nos cages expérimentales et nous tenterons d'en déterminer les causes.

\section{$3^{\circ}$ Cages Benton, reines jeunes, accompagnées de 20 ouvrières, sans apport d'eau.}

Le nombre d'abeilles réunies dans les cages de cette série est bien proche du maximum possible; il faut, en effet, que les insectes puissent continuer à se déplacer assez librement. La comparaison avec les témoins de la série I indique des résultats légèrement meilleurs puisqu'on trouve une durée de vie moyenne de I9, I jours avec des extrêmes de 15 et de 25 jours. Le chiffre de 10 abeilles que nous utilisons dans les autres séries en cages Benton est donc loin d'être un maximum car son augmentation ne réduit absolument pas la durée de survie des abeilles dans les cages.

\section{$4^{\circ}$ Cages expérimentales, reines jeunes, accompagnées de 40 à 50 ouvrières sans apport d'eau}

Dans ces cages, les ouvrières circulent très librement sans toutefois disposer d'un trop grand espace. Le tableau I montre que nous obtenons ici les meilleurs résultats de l'ensemble de nos essais avec ce type de cage et ce nombre d'abeilles: 35,2 jours de moyenne de vie (minimum 23 jours, maximum 47 jours). Ces excellents résultats semblent être dus à un bon rapport entre le volume de la cage et le nombre d'abeilles qui la peuple. On y trouve sans doute réunies les meilleures conditions de vie possible dans un petit volume. Les nécessités physiologiques de l'abeille y sont largement satisfaites.

\section{$5^{\circ}$ Cages expérimentales, reines jeunes,} accompagnées de 40 à 50 ouvrières, avec apport d'eau

Les conditions de vie dans cette série sont les mêmes que dans la précédente, a seule différence étant l'apport d'eau. Nous avons dit déjà, à propos de la série 2 , notre surprise de voir les abeilles abreuvées mourir avant les abeilles sans eau. Nous retrouvons ici des résultats analogues. Bien que la moyenne de vie $(26,5$ jours $)$ soit inférieure de 8,7 jours à celle de la série précédente (sans eau), ce résultat vient en deuxième position pour l'ensemble de nos essais.

Il semble que la réduction de la durée de vie sous l'action de l'apport d'eau soit explicable. Dans les travaux que nous avons précédemment cités, l'eau est toujours distribuée dans des abreuvoirs spécialement conçus à cet effet. I'orifice qui permet aux abeilles de boire est très petit ; ainsi, l'évaporation est minime et le pourcentage d'humidité de l'air à l'intérieur de la cage n'est pas augmenté. En outre, les expériences étant conduites dans des étuves, le pourcentage d'humidité dans l'air était réglé automatiquement. Par contre, dans nos essais, le coton imbibé d'eau couvre une partie du grillage d'aération de la cage. Bien qu'aucune goutte de liquide n'ait coulé dans les cages, l'évaporation a pu augmenter le pourcentage d'humidité dans l'air des cages et nuire ainsi à la longévité de leurs occupantes. Le refroidissement 


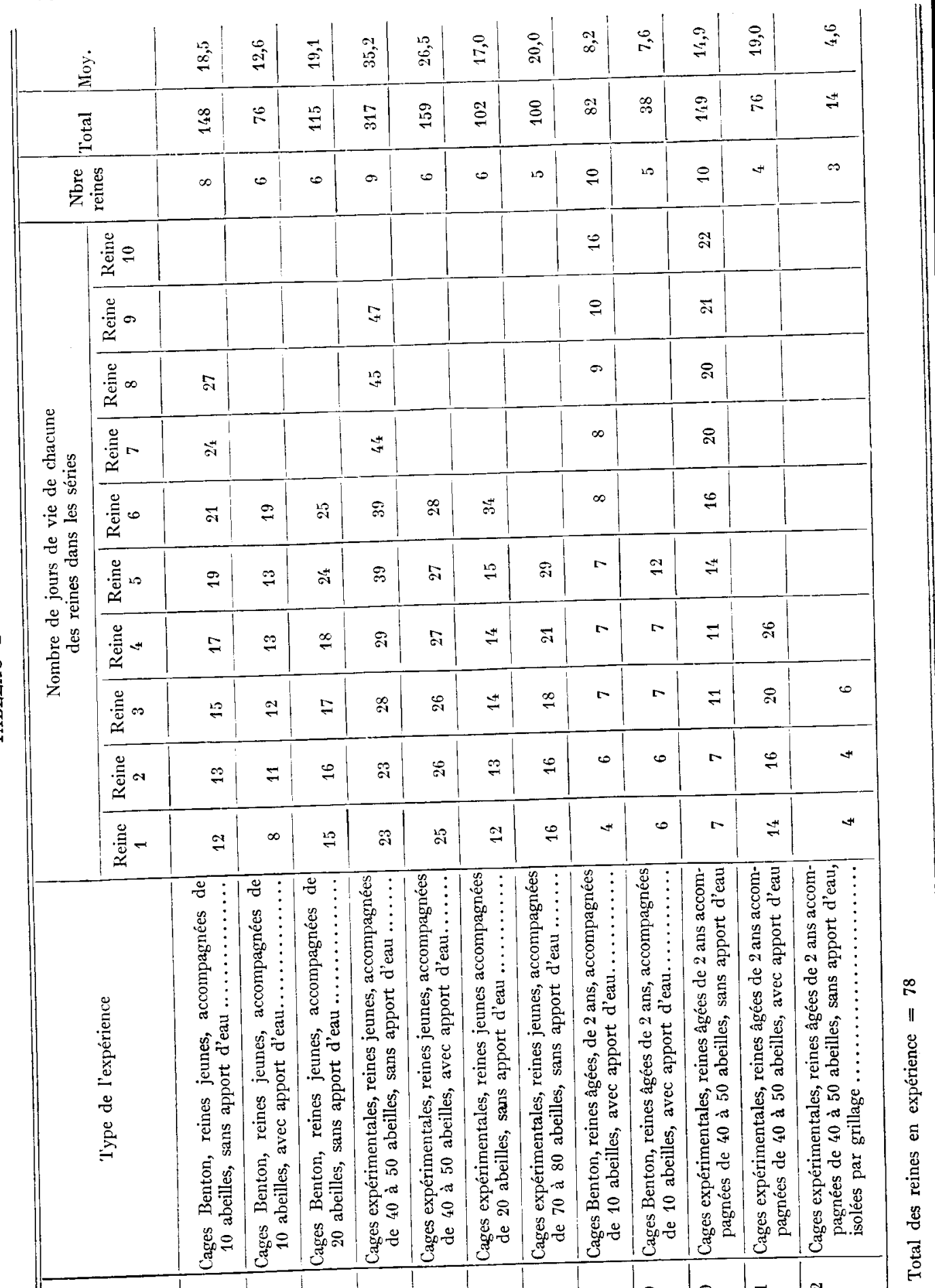


provoqué par l'évaporation de cette eau peut également être mis en cause. Mrais un autre facteur, qui ne serait pas à imputer à la façon de distribuer l'eau mais à l'eau elle-même, peut être invoqué. Nous avons été frappé par la différence de propreté qui existe dans les cages contenant des abeilles abreuvées et les autres. Les cages contenant des abeilles non abreuvées restent propres, sans déjections; au contraire, les autres sont très souillées d'excréments. Les abeilles, qu'elles soient vivantes ou à l'état de cadavres, deviennent noires et gluantes, alors que celles des cages sans eau restent sèches et propres. L'ampoule rectale des ouvrières abreuvées est gonflée d'excréments liquides. Il semble que ces abeilles consomment trop de candi. L'eau leur permet de le diluer sans difficulté. Par contre, les abeilles des cages sans eau sont soumises à une diète partielle du fait qu'elles ne peuvent consommer le candi que très lentement; de ce fait, elles vivent plus longtemps.

\section{$6^{\circ}$ Cages expérimentales, reines jeunes, accompagnées de 20 ouvrières, sans apport d'eau}

Cette série est à comparer à la série 3. Ici, c'est l'influence du volume, pour un même nombre d'abeilles, qui est testée. I a moyenne de durée de vie est de I7 jours (I2 à 34 jours). Ce maximum de 34 jours fausse notablement la moyenne. En effet la reine $n^{0} 6$ constitue un cas unique au cours de nos expériences. Elle a réussi à vivre 7 jours après que la dernière abeille de la cage soit morte, ce qui ne se produit jamais. On peut se demander ce qui a pu lui permettre une telle survie. Ia moyenne se situerait plus normalement à I4 jours environ. On note un raccourcissement très net de la durée de vie par rapport à la série 3 , raccourcissement qui peut etre imputé à une occupation insuffisante de la cage, donc à une densité trop faible d'abeilles pour le volume disponible. Nous retrouvons, quoique beaucoup moins marquée, la différence existant entre les séries I et 3 .

$7^{\circ}$ Cages expérimentales, reines jeunes, accompagnées de 70 à 80 abeilles, sans apport d'ean

Nous testons ici la densité maximum d'abeilles dans les cages expérimentales. Cette série est également à comparer aux séries 3,4 et 6 . La durée moyenne de vie de 20 jours ( 16 à 29 jours), lui donne la troisième place dans nos essais. On peut supposer que le nombre d'abeilles y favorise une longue durée de vie mais que l'optimum de densité de population est dépassé.

$8^{\circ}$ Cages Benton, reines âgées de 2 ans, accompagnées de 10 ouvrières, sans apport d'eau

Bien que les reines âgées soient beaucoup plus rarement expédiées en cages de transport que les reines jeunes, il nous a semblé intéressant de connaittre leurs possibilités de survie dans les mêmes conditions. Les résultats de cette série et des suivantes seront cependant moins précis que ceux des essais sur les reines jeunes car nu facteur très important, négligeable chez les reines jetınes, s'ajoute dans le cas des reines âgées. Il s'agit de la grande variabilité naturelle de la durée de vie des 
reines. Ainsi, dans cette série, le maximum de vie de 16 jours est quadruple du minimum, 4 jours. La moyenne de vie est de 8,2 jours. Comparé à celui de la série $I$, ce résultat montre une réduction de plus de $5^{\circ} \mathrm{p}$. Ioo des possibilités de survie chez les reines âgées de 2 ans.

\section{$9^{\circ}$ Cages Benton, reines âgées de 2 ans, accompagnées de 10 ouvrières avec apport d'eau}

Cette série est semblable à la série 2 mais avec des reines âgées au lieu de reines jeunes. Comparée à la série 8 , on constate un raccourcissement de la durée de survie provoqué par l'apport d'eau, ce qui confirme les résultats obtenus dans les précédentes séries. La durée moyenne de vie est de 7,6 jours ( 6 à 12 jours).

I0 ${ }^{\circ}$ Cages expérimentales, reines âgées de 2 ans, accompagnées de 40 à 50 ouvrières, sans apport d'eau

C'est la répétition, avec des reines âgées, de la série 4 . Comparée à la série $S$ en cages Benton, on retrouve la même augmentation de durée de vie due au nombre plus important d'ouvrières qui accompagnent les reines. La durée de vie moyenne est de 14,9 jours ( 7 à 22 jours).

II Cages expérimentales, reines âgées de 2 ans, accompagnées de 40 à 50 ouvrières, avec apport d'ean

C'est la répétition, avec des reines âgées, de la série 5 . Comparée à la série ro, cette série montre une amélioration de la durée de vie ; I9 jours de moyenne ( 14 à 20 jours). Il semblerait que les reines âgées aient un plus grand besoin d'eau que les jeunes reines. Cependant, l'interprétation des résultats est plus délicate dans le cas des reines âgées en raison de la variabilité naturelle de leurs possibilités de survie.

\section{I $2^{\circ}$ Cages expérimentales, reines âgées de 2 ans, isolées par un grillage accompagnées de 40 à 50 ouvrières, sans apport d'eau}

Les reines sont isolées par un grillage à mailles de $2,5 \mathrm{~mm}$. Cette série a été ajoutée dans le but de tenter de concilier les difficultés de l'introduction des reines et les problèmes de transport. La cage intérieure en grillage qui contient la reine seule peut être retirée de la cage de transport sans risque de la blesser ou de la perdre. Son très petit volume lui permet de servir ensuite de cage d'introduction.

Les très mauvais résultats obtenus au cours des premiers essais nous ont rapidement fait abandonner cette tentative. 3 reines ainsi emprisonnées donnèrent une durée moyenne de vie de 4,6 jours. Une seconde raison a provoqué 1'abandon de cette méthode : le peu de chances de vulgarisation d'un type de cage forcément complexe et d'un prix de revient élevé. Eille était peut être perfectible cependant, car SEBEsr (I957) et GRIFFin (I963) ont fait hiverner des reines isolées dans des cages grillagées, mais dans des groupes d'abeilles beaucoup plus importants. FrEE et BUTLER (1958) ont montré que la grandeur des ouvertures à travers lesquelles les abeilles pouvaient échanger de la nourriture étaient au minimum de $2,5 \mathrm{~mm}$. 


\section{OBSERVATIONS SUR IA CONSOMMATION DU CANDI}

Le volume du magasin destiné au candi dans nos cages expérimentales est de I6 $\mathrm{cm}^{3}$ environ, ce qui représente un poids approximatif le $23 \mathrm{~g}$ de provisions. Ces $23 \mathrm{~g}$ de candi suffisent à la nourriture des 40 à 50 abeilles de nos cages expérimentales pendant une durée de I4 à I6 jours. La nourriture journalière pour un tel groupe d'abeilles est donc d'environ $\mathrm{I}, 5 \mathrm{~g}$, soit $0,033 \mathrm{~g}$ par abeille, ou encore, $0,5 \mathrm{~g}$ pour une abeille pendant I5 jours.

Dans les conditions extrêmes de nos expériences, allant jusqu'à la mort des abeilles et des reines, nous étions dans l'obligation de renouveler la réserve de candi avant son épuisement total. Il est absolument indispensable qu'il ne manque jamais de candi dans les cages, car les reines, qui meurent habituellement les dernières ou presque, meurent régulièrement dans les 24 heures qui suivent le manque de nourriture. Par contre, un certain nombre d'abeilles survivent quelques jours à la reine. Il semble donc que les abeilles abandonnent la reine et cessent de la nourrir dès que la nourriture est épuisée et que celle-ci résiste moins bien que les ouvrières à la famine. Nous n'arons pas jugé utile d'augmenter le volume du magasin destiné au candi dans nos cages expérimentales car, dans les conditions normales de transport de reines, il n'y a pratiquement pas de risques que l'emprisonnement de la reine atteigne $I_{5}$ jours.

\section{DISCUSSION}

Les expériences dont les résultats sont présentés dans le tableau I démontrent une très nette supériorité des cages expérimentales que nous avons décrites sur les cages de transport de reines habituellement utilisées.

La durée moyenne de vie des reines dans nos cages atteint 35,2 jours clans l'expérience $\mathbf{n}^{\circ}+$ qui réunit les meilleures conditions : 40 à 50 abeilles par cage, volume convenable de cette cage, pas d'apport d'eau. Lres reines de la série no 5 atteignent une durée moyenne de vie de 26,5 jours dans les mêmes conditions que la série $n^{\circ} 4$ mais avec, en outre, un apport d'eau à l'aide de coton hydrophile. L'eau ainsi apportée est nuisible aux abeilles puisqu'elle cause une réduction de la durée moyenne de vie de 8,7 jours, qui provient probablement de l'augmentation du taux d'humidité de l'air de la cage, du froid occasionné par l'évaporation de cette eau ainsi que, principalement, du gonflement anormal de l'ampoule rectale dî à une consommation trop importante de nourriture.

Les reines accompagnées de Io abeilles dans les cages Benton ont une durée moyenne de vie de $I 8,5$ jours. La différence avec les reines accompagnées de 40 à 50 abeilles atteint $I 6,7$ jours. Il est donc indiscutable que l'influence du nombre d'abeilles d'accompagnement est primordiale en ce qui concerne la durée de vie des reines dans les cages d'expédition. 
La figure 2, qui groupe les résultats obtenus avec les cages expérimentales dans les séries 4 et 5 d'une part, et ceux obtenus avec les cages Benton dans les séries I et 2 d'autre part, illustre bien nos résultats partiels.

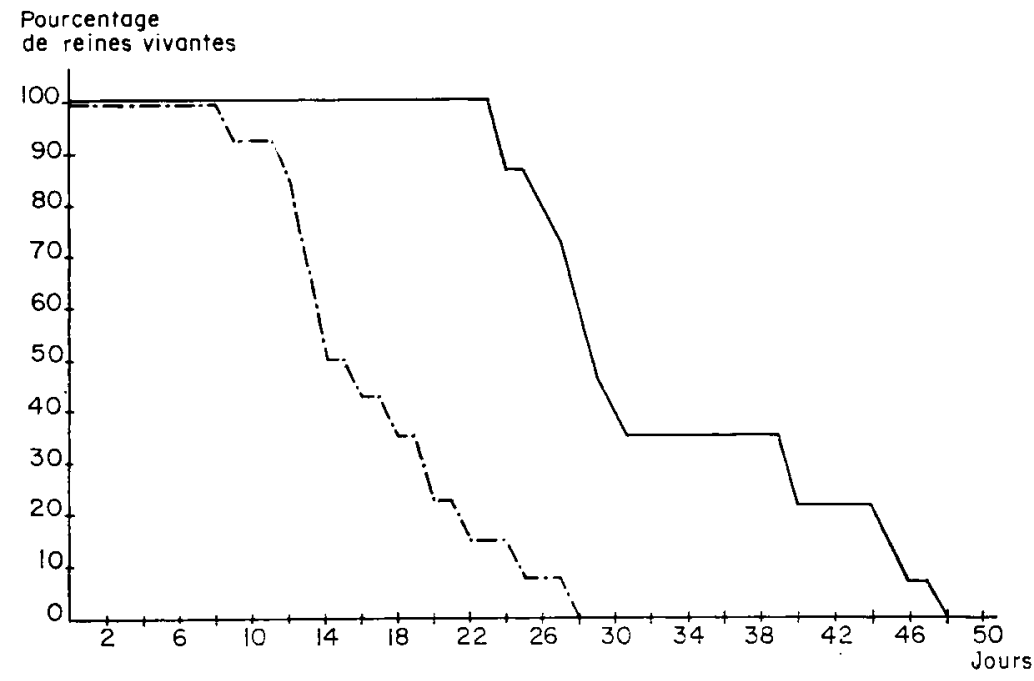

FIG. 2. - Pourcentage des reines survivantes en fonction dil temps

Trait plein $=$ Séries $4+5$

Trait pointille $=$ Séries $\mathrm{I}_{-}+2$

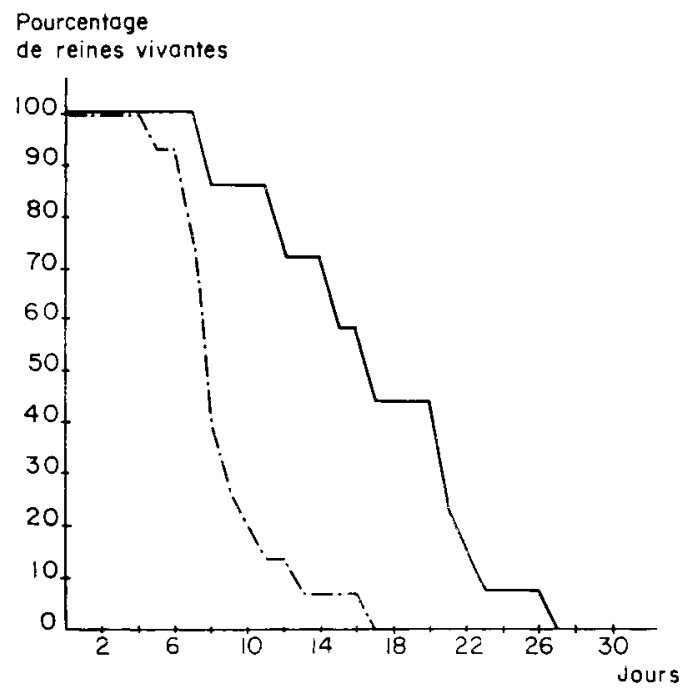

FIG. 3. - Pourcentage des reines survivantes en fonction du temps

Trait plein $=$ Séries Io + I I

Trait pointillé $=$ Séries $8+9$

Les reines âgées de 2 ans des séries ro et II comparées à celles des séries 8 et 9 (fig. 3) fournissent des résultats en accord avec notre thèse puisque nous trou- 
vons $\mathrm{I}_{4,9} 9$ et $\mathrm{I} 9$ jours de survie pour les reines accompagnées de 40 à 50 abeilles contre seulement 8,2 et 7,6 jours de vie pour les reines accompagnées de ro abeilles. Les séries groupées dans les graphiques des figures 2 et 3 permettent d'établir des moyennes sur de plus grands nombres. On trouve, pour les reines jeunes en cages expérimentales, tune moyenne de survie de $3 x, 73$ jours, et pour les reines jeunes en cages Benton, de $I 6,0$ jours. Pour les reines âgées de 2 ans, la moyenne, dans les cages expérimentales, est de I6,07 jours ; dans les cages Benton, elle est de 8,0 jours. Ainsi, la durée de survie des reines est presque doublée dans les cages expérimentales comparativement aux cages Benton, et cela aussi bien pour les reines jeunes que pour les reines âgées de 2 ans.

Les reines âgées de 2 ans ont une durée de vie beaucoup plus courte que les reines jeunes, lorsqu'elles sont en cages, mais les cas de transport de reines de cet âge sont extrêmement rares dans la pratique.

La série 3 (Ig, I jours de durée moyenne de vie, cages Benton avec ro abeilles), comparée à la série I ( 18,5 jours de durée moyenne de vie), est également en faveur de l'augmentation du nombre d'abeilles. Comparée à la série 6 ( $\mathrm{I} 7$ jours de durée moyenne de vie), elle démontre que le volume de la cage doit être proportionnel au nombre d'abeilles et que, dans ce dernier cas, les abeilles sont trop peu nombreuses pour nos cages expérimentales. A l'opposé, la série 7 (20 jours de durée moyenne de vie), indique que le nombre optimum d'abeilles pour nos cages est dépassé. Il est vraisemblable que, puisque nos cages expérimentales utilisées dans les conditions de la série 4 , donnent les meilleurs résultats, une augmentation proportionnelle du volume de la cage, de la nourriture, et du nombre d'abeilles améliorerait encore la durée de vie des reines. Mais il faut considérer la destination de ces cages. Elles doivent permettre le transport des reines dans de bonnes conditions, de façon pratique, et à peu de frais. Dans ces conditions, il semble que les proportions que nous avons retenues soient les plus raisonnables.

I'amélioration de la durée de vie que nous avons obtenue est telle qu'il est vraisemblable qu'elle corresponde à un plus grand respect des besoins physiologiques des reines sur le plan des échanges de nourriture, de la production de chaleur et de l'effet de groupe. Les travaux antérieurs nous confirment d'ailleurs dans cette idée qu'il est important de dépasser le seuil que représente le nombre de trente individus environ à partir duquel la survie est nettement favorisée par rapport à ce qui se passe dans les groupes numériquement plus faibles.

Il est bien évident qu'en soi la durée de vie maximum des reines en cages d'expédition ne présente que peu d'intérêt sur le plan pratique, car on s'efforce toujours de réduire le plus possible la durée des transports. Il est rare que celle-ci dépasse trois jours dans les conditions du trafic postal intérieur et cinq jours dans les conditions du trafic international. Cependant, on est en droit de se demander si 1'augmentation de la durée de survie que nous avons réussi à obtenir ne peut pas être considérée comme un facteur susceptible d'être lié aux facilités d'acceptation des reines au moment de leur introduction, puis à la reprise de la ponte. Rappelons à ce propos le travail de DREHER (I954), précédemment cité, qui a mis en évidence l'importance de la durée de la claustration sur le comportement ultérieur de la reine. Il serait donc intéressant de reprendre ce travail en considérant le cas de reines encagées dans des conditions plus favorables que celles qui sont habituellement réunies. En augmentant sensiblement le nombre des abeilles d'accompagnement, le volume de 
la cage et la quantité de nourriture disponible, on est en droit de penser qu'il y aurait une amélioration sensible du " confort physiologique " des reines claustrées, amélioration susceptible de modifier les conclusions du travail de DREHER.

Les recherches devraient donc se poursuivre maintenant dans ce sens et, dans le cas où un résultat positif serait obtenu, il conviendrait de revoir la question des conditions d'utilisation des cages du modèle que nous avons mis au point. En effet, ces cages ne sont pas adaptées à l'introduction directe des reines; par ailleurs, une augmentation du nombre des abeilles d'accompagnement ne va pas sens soulever bon nombre de difficultés a11 moment de l'introduction.

Nous nous proposons donc maintenant d'étudier l'influence des conditions de claustration sur l'acceptation des reines puis sur la reprise de la ponte ainsi que sur les accidents de supersédure. C'est en fonction des résultats obtenus que nous pourrons envisager de revoir complètement les techniques de transport des reines sur de longtres distances.

Reçu pour publication en avril 1965.

\section{SUMMARY}

THF LIFE-LENGTH OF TIE QIEEN IIONEYIBES «APIS MELLIFICA MELLIFICA IN EXPEDITION CAGES

Experiences have been carried out to study the influence of the number of escorting bees on the length of life of the queens in expedition cages.

An increase in the number of these working-bees, 40 to 50 in the experimental cages against 5 to 10 in the expedition cages of the Benton-type, allows to double the avcrage length of the life of queens in cages.

We showed the importance of the cage-volume, which should be proportional to the number of bees in it, as well as the influence of different factors such as food and hygrometry.

\section{RÉFÉRENCES BIBLIOGRAPHIQUES}

Al.fex XI. 1), 1955. Observations on honeybees attending their queen. Brit. J. Anim. Behaviour, 3 (2), 66-69.

Cinclin R., 195I. Sur le déterminisme de l'effet de groupe chez les abeilles. Physiol. comp. Qicol., $282-288$.

Citalvin R., Grasse P'. l'., ig)44. J'effet de groupe et la survie des neutres dans les sociétés animales. Rez. Scient., 182, 465-464.

Drfiner K., 1954. Ueber den lïnfluss längerer Ilaft im Versandkäfig auf begattete Jungköniginnen. Hessische Biene., $90(3), 81-83$.

FrEE J. B., ButLer C. (i., 1958. 'The size of apertures through which worker honeybees will feed one another. Bee World, 39 (2), 40-42.

FREE J. B., SPENCRR BOoth Y., In59. The longevity of worker honey bees (Apis mellifera). Roy. Entom. Soc. London, 34 (1 2 ), $14^{1-1}, 0$.

Griffix L. A. II.. ig6.3. Improved cages and management for queen bee bank system. Nerc \%eal. I. agric. Res., $106(\%), 287-289$.

KuARchis A. I. T957. Variations du poids des reines maintenues en cages avec des ouvrieres (en russe).

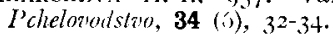

LAvie l'., RoTiI M., 1953. Sur le thermopréférendum et la production de chaleur chez les abeilles. Physiol. Comp. (Ecol., 3 (I), 5i-62.

MaURizio A., 1946. Beobachtungen über die Lebensdauer und den Futterbrauch gefangen gehaltener Jienen. Beil. Schrieiz. Bienent., $13,1-+8$.

Morse R. A., Gary N. J., ighi. Colnny response to worker bees confined with queens (Apis mellifera L..). Bee World, 42 (8), 197-199. 
Orosi-PAL Z., 1955. Rôle des abeilles accompagnatrices dans l'acceptation des reines (en hongrois). Meheszet, 3 (I I), 203-207.

Rocissteis M., 1950. Longevity in the adult worker honeybee. Ann. Enlomol. Soc. Amer., 43 (I), I52-I54. SERESt K., 1957. Hivernage des reines de réserve (en hongrois). .leheszet, 5 (10), I87-r88.

SNeLGRove L. E., 1943. The introduction of queen Bees. Purwell and Sons, London, $3^{3} e$ éd.

Woonkow A. W., 1938. Equipement and procedure for longevity studies with caged honeybees. U.S.D. A. Ent. Plant. Quar. E., 438, p. 6.

Woonrow A. W., I94 I. Some effects of temperature, relative humidity, confinement, and type of food on queen bees in mailing cages. U. S. D. A. Ent. Plant. Quar., E, 529, p. I2. 